Sains Malaysiana 50(12)(2021): 3745-3751

http://doi.org/10.17576/jsm-2021-5012-23

\title{
Toeplitz Determinant for a Subclass of Tilted Starlike Functions with Respect to Conjugate Points
}

(Penentu Toeplitz untuk Subkelas Fungsi Bak Bintang Miring terhadap Titik-titik Konjugat)

\author{
Nur HaZWANi AqILAH AbDul WaHid \& DAUd MOHAMaD*
}

\section{ABSTRACT}

Let $S_{c}^{*}(\alpha, \delta, A, B)$ denote the class of analytic and univalent functions in an open unit disk $E=\{z \in \mathbb{C}:|z|<1\}$ of the form $f(z)=z+\sum_{n=2}^{\infty} a_{n} z^{n}$ and satisfy $\left\{e^{i \alpha} \frac{z f^{\prime}(z)}{g(z)}-\delta-i \sin \alpha\right\} \frac{1}{t_{\alpha \delta}} \prec \frac{1+A z}{1+B z}$ where $g(z)=\frac{f(z)+\overline{f(\bar{z})}}{2}, t_{\alpha \delta}=\cos \alpha-\delta>0$, $0 \leq \delta<1,|\alpha|<\frac{\pi}{2}$ and $-1 \leq B<A \leq 1$. This paper presents the coefficient bounds for functions in $S_{c}^{*}(\alpha, \delta, A, B)$ using symmetric Toeplitz determinants $T_{2}(2), T_{3}(2)$ and $T_{3}(1)$. The results obtained generalize the results for some existing subclasses in the literature.

Keywords: Coefficient bounds; starlike functions with respect to conjugate points; Toeplitz determinant

\section{ABSTRAK}

Andaikan $S_{c}^{*}(\alpha, \delta, A, B)$ sebagai kelas fungsi analisaan dan univalen dalam cakera unit terbuka $E=\{z \in \mathbb{C}:|z|<1\}$ dalam bentuk $f(z)=z+\sum_{n=2}^{\infty} a_{n} z^{n}$ dan memenuhi syarat $\left\{e^{i \alpha} \frac{z f^{\prime}(z)}{g(z)}-\delta-i \sin \alpha\right\} \frac{1}{t_{\alpha \delta}} \prec \frac{1+A z}{1+B z} \operatorname{dengan} g(z)=\frac{f(z)+\overline{f(\bar{z})}}{2}$, $t_{\alpha \delta}=\cos \alpha-\delta>0,0 \leq \delta<1,|\alpha|<\frac{\pi}{2}$ dan $-1 \leq B<A \leq 1$. Makalah ini membentangkan batas-batas pekali bagi fungsi dalam $S_{c}^{*}(\alpha, \delta, A, B)$ menggunakan penentu-penentu Toeplitz $T_{2}(2), T_{3}(2)$ dan $T_{3}(1)$. Keputusan yang diperoleh mengitlak keputusan beberapa subkelas dalam kajian lepas.

Kata kunci: Batas-batas pekali; fungsi bak bintang terhadap titik-titik konjugat; penentu Toeplitz

\section{INTRODUCTION}

Let $H$ be the class of functions $\omega$ which are analytic in an open unit disk $E=\{z \in \mathbb{C}:|z|<1\}$ given by

$$
\omega(z)=\sum_{k=2}^{\infty} b_{k} z^{k}
$$

and satisfying the conditions $\omega(0)=0,|\omega(z)|<1, z \in E$. Let two functions $F(z)$ and $G(z)$ be analytic in $E$. If there exists a Schwarz function $\omega \in H$ which is analytic in $E$ such that $F(z)=G(\omega(z))$, then $F(z) \prec G(z)$. The symbol " $\prec$ " denotes the subordination. Further, if $G(z)$ is univalent in $E$, then $F(z) \prec G(z) \Leftrightarrow F(0)=G(0)$ and $F(E)=G(E)$.

Let $\mathrm{A}$ be the class of functions $f$ which are analytic and univalent in $E$ and of the form

$$
f(z)=z+\sum_{n=2}^{\infty} a_{n} z^{n}, z \in E .
$$

We denote by $S$ the subclass of A consisting of all univalent functions in $E$. The well-known subclasses of $S$ namely starlike, convex and close-to-convex functions, respectively denoted by $S^{*}, K$ and $C$.

In 1987, El-Ashwah and Thomas defined the class of starlike functions with respect to conjugate points $S_{C}{ }^{*}$ consisting of functions of the form (2) and satisfying the condition

$$
\operatorname{Re}\left\{\frac{2 z f^{\prime}(z)}{f(z)+\overline{f(\bar{z})}}\right\}>0, z \in E
$$

In 1991, Halim defined the class $S_{C}{ }^{*}(\delta)$ consisting of functions of the form (2) and satisfying the condition 


$$
\operatorname{Re}\left\{\frac{2 z f^{\prime}(z)}{f(z)+\overline{f(\bar{z})}}\right\}>\delta, 0 \leq \delta<1, z \in E
$$

In 2009, Dahhar and Janteng introduced the class $S_{C}{ }^{*}(A$, $B$ ) consisting of functions of the form (2) and satisfying the condition

$$
\frac{2 z f^{\prime}(z)}{f(z)+\overline{f(\bar{z})}} \prec \frac{1+A z}{1+B z},-1 \leq B<A \leq 1, z \in E .
$$

By definition of subordination, from (5), it follows that $f \in S_{C}{ }^{*}(A, B)$ if and only if

$$
\frac{2 z f^{\prime}(z)}{f(z)+\overline{f(\bar{z})}}=\frac{1+A \omega(z)}{1+B \omega(z)}, \omega \in H .
$$

Motivated from the work of El-Ashwah and Thomas (1987), Halim (1991) and Dahhar and Janteng (2009), Wahid et al. (2015) introduced the subclass of tilted starlike functions with respect to conjugate points of order $\delta S_{C}{ }^{*}(\alpha, \delta, A, B)$ consisting of functions given by (2) and satisfying the condition

$$
\left\{e^{i \alpha} \frac{z f^{\prime}(z)}{g(z)}-\delta-i \sin \alpha\right\} \frac{1}{t_{\alpha \delta}} \prec \frac{1+A z}{1+B z},
$$

where $g(z)=\frac{f(z)+\overline{f(\bar{z})}}{2}, t_{\alpha \delta}=\cos \alpha-\delta>0,0 \leq \delta<1$, $|\alpha|<\frac{\pi}{2}$ and $-1 \leq B<A \leq 1$.

By definition of subordination, from (7), it follows that $f \in S_{C}{ }^{*}(\alpha, \delta, A, B)$ if and only if

$$
\left\{e^{i \alpha} \frac{z f^{\prime}(z)}{g(z)}-\delta-i \sin \alpha\right\} \frac{1}{t_{\alpha \delta}}=\frac{1+A \omega(z)}{1+B \omega(z)}, \omega \in H .
$$

Obviously, the functions in these classes $S_{C}{ }^{*}(\delta)$, $S_{C}{ }^{*}(A, B)$ and $S_{C}{ }^{*}(\alpha, \delta, A, B)$, respectively, are the subclass of the classes $S_{C}{ }^{*}=S_{C}(0), S_{C}{ }^{*}=S_{C}{ }^{*}(1,-1)$ and $S_{C}{ }^{*}=S_{C}{ }^{*}(0,0,1,-1)$.

It is known that the Toeplitz matrices are closely related to the Hankel matrices and one of the well-studied classes of structured matrices. The Toeplitz matrices have constant entries along the diagonals whereas the Hankel matrices have constant entries along the reverse diagonals. The Toeplitz matrices have many applications in the branches of pure and applied mathematics that led to some of the major developments of studies related to Toeplitz determinants, Toeplitz kernel, Toeplitz operators and $q$-deformed Toeplitz matrices (see Ye and Lim (2016) for details). Recall that the Hankel determinant $H_{q}(n), n, q \geq 1$ for $f$ with the form as in (2) was defined by Pommerenke (1966) and Pommerenke (1967) as

$$
H_{q}(n)=\left|\begin{array}{cccc}
a_{n} & a_{n+1} & \cdots & a_{n+q-1} \\
a_{n+1} & a_{n+2} & \cdots & a_{n+q} \\
\vdots & \vdots & \vdots & \vdots \\
a_{n+q-1} & a_{n+q} & \cdots & a_{n+2 q-2}
\end{array}\right| .
$$

Thomas and Halim (2016) introduced the symmetric Toeplitz determinant $T_{q}(n), n, q \geq 1$ for $f$ with the form as in (2) given by

$$
T_{q}(n)=\left|\begin{array}{cccc}
a_{n} & a_{n+1} & \cdots & a_{n+q-1} \\
a_{n+1} & a_{n} & \cdots & a_{n+q-2} \\
\vdots & \vdots & \vdots & \vdots \\
a_{n+q-1} & a_{n+q-2} & \cdots & a_{n}
\end{array}\right|, a_{1}=1 .
$$

In particular, we have

$$
\begin{aligned}
T_{2}(2) & =\left|\begin{array}{ll}
a_{2} & a_{3} \\
a_{3} & a_{2}
\end{array}\right|=a_{2}{ }^{2}-a_{3}{ }^{2}, \\
T_{3}(1) & =\left|\begin{array}{ccc}
1 & a_{2} & a_{3} \\
a_{2} & 1 & a_{2} \\
a_{3} & a_{2} & 1
\end{array}\right|=1\left(1-a_{2}{ }^{2}\right)-a_{2}\left(a_{2}-a_{2} a_{3}\right)+a_{3}\left(a_{2}{ }^{2}-a_{3}\right), \\
T_{3}(2) & =\left|\begin{array}{lll}
a_{2} & a_{3} & a_{4} \\
a_{3} & a_{2} & a_{3} \\
a_{4} & a_{2} & a_{2}
\end{array}\right|=a_{2}\left(a_{2}{ }^{2}-a_{2} a_{3}\right)-a_{3}\left(a_{2} a_{3}-a_{3} a_{4}\right)+ \\
& a_{4}\left(a_{2} a_{3}-a_{2} a_{4}\right),
\end{aligned}
$$

and so on.

In recent years, the studies in a problem of estimating the coefficient bounds for the Toeplitz determinants for the class of univalent functions and its subclasses have been done by some researchers such as Al-Khafaji et al. (2020), Ali et al. (2018), Radhika et al. (2018, 2016), Ramachandran and Kavitha (2017), Sivasubramanian et al. (2016), Srivastava et al. (2019), Thomas and Halim (2016), and Zhang et al. (2019). However, there was no study of finding estimates for the Toeplitz determinants $T_{2}(2), T_{3}(2)$ and $T_{3}(1)$ for the subclasses introduced by El-Ashwah and Thomas (1987), Halim (1991) and Dahhar and Janteng (2009). In this paper, we determine the coefficient bounds for the Toeplitz determinants $T_{2}(2)$, $T_{3}(1)$ and $T_{3}(2)$ for the class $S_{C}{ }^{*}(\alpha, \delta, A, B)$ consisting the functions given by (2). We also give some results for the subclasses introduced by El-Ashwah and Thomas (1987), Halim (1991) and Dahhar and Janteng (2009).

We shall state the following lemmas to prove our main results. 


\section{PRELIMINARY RESULTS}

Let $P$ be the class of functions $p$ of the form

$$
p(z)=1+\sum_{n=1}^{\infty} p_{n} z^{n}
$$

that is analytic in $E$ and satisfying the condition $\operatorname{Re} p(z)>0, z \in E$. Functions in $P$ are sometimes called Carathéodory functions.

Lemma 1 (Duren 1983) (p. 41) For a function $p \in P$ of the form (9), the sharp inequality $\left|p_{n}\right| \leq 2$ holds for each $n \geq 1$. Equality holds for the function $p(z)=\frac{1+z}{1-z}$.

Lemma 2 (Efraimidis 2016) Let $p \in P$ of the form (9) and $\mu \in \mathbb{C}$. Then

$$
\left|p_{n}-\mu p_{k} p_{n-k}\right| \leq 2 \max \{1,|2 \mu-1|\}, 1 \leq k \leq n-1 .
$$

If $|2 \mu-1| \geq 1$, then the inequality is sharp for the function $p(z)=\frac{1+z}{1-z}$ or its rotations. If $|2 \mu-1|<1$, then the inequality is sharp for the function $p(z)=\frac{1+z^{n}}{1-z^{n}}$ or its
rotations.

\section{MAIN RESULTS}

Theorem 1 If $f \in S_{C}{ }^{*}(\alpha, \delta, A, B)$, then

$$
\begin{aligned}
\left|a_{2}{ }^{2}-a_{3}\right| & \leq \frac{T^{2}}{64}\left\{64+16 \sqrt{\left[2(1+B) T-\cos \alpha\left((1+B)^{2}+T^{2}\right)\right]^{2}+\left(-\sin \alpha\left((1+B)^{2}-T^{2}\right)\right)^{2}}\right. \\
& \left.+16 \sqrt{(1+2 B)^{2}-4(1+2 B) T \cos \alpha+4 T^{2}}\right\}
\end{aligned}
$$

where $T=(A-B) t_{\alpha \delta}$ and $t_{\alpha \delta}=\cos \alpha-\delta$. The inequality is sharp.

Proof. As $f \in S_{C}^{*}(\alpha, \delta, A, B)$, so from (8)

$$
\left\{e^{i \alpha} \frac{z f^{\prime}(z)}{g(z)}-\delta-i \sin \alpha\right\} \frac{1}{t_{\alpha \delta}}=\frac{1+A \omega(z)}{1+B \omega(z)}, \omega \in H
$$

where $g(z)=\frac{f(z)+\overline{f(\bar{z})}}{2}$ and $t_{\alpha \delta}=\cos \alpha-\delta$.

Now, let

$$
h(z)=\frac{1+\omega(z)}{1-\omega(z)}=1+\sum_{n=1}^{\infty} k_{n} z^{n} .
$$

We have $h \in P$ and

$$
\omega(z)=\frac{h(z)-1}{h(z)+1} .
$$

Thus, by using (11), from (10), we have

$$
e^{i \alpha} \frac{z f^{\prime}(z)}{g(z)}=\frac{\left[e^{i \alpha}(1-B)-T\right]+h(z)\left[e^{i \alpha}(1+B)+T\right]}{1-B+h(z)(1+B)}
$$

where $T=(A-B) t_{\alpha \delta}$.

Using the series expansion in (12), we get

$e^{i \alpha}(1-B)\left(z+2 a_{2} z^{2}+3 a_{3} z^{3}+\cdots\right)$

$$
\begin{aligned}
& +e^{i \alpha}(1+B)\left(z+2 a_{2} z^{2}+3 a_{3} z^{3}+\cdots\right)\left(1+k_{1} z+k_{2} z^{2}+\cdots\right) \\
= & {\left[e^{i \alpha}(1-B)-T\right]\left(z+a_{2} z^{2}+a_{3} z^{3}+\cdots\right) } \\
& +\left[e^{i \alpha}(1+B)+T\right]\left(z+a_{2} z^{2}+a_{3} z^{3}+\cdots\right)\left(1+k_{1} z+k_{2} z^{2}+\cdots\right) .
\end{aligned}
$$

Equating the coefficients of $z^{2}, z^{3}$ and $z^{4}$ in the expansion of (13) give us

$$
\begin{gathered}
a_{2}=\frac{k_{1} T e^{-i \alpha}}{2}, \\
a_{3}=\frac{2 k_{2} T e^{-i \alpha}+k_{1}^{2} T^{2} e^{-2 i \alpha}-(1+B) k_{1}^{2} T e^{-i \alpha}}{8},
\end{gathered}
$$

and

$$
\begin{aligned}
a_{4}= & \frac{8 k_{3} T e^{-i \alpha}+6 k_{1} k_{2} T^{2} e^{-2 i \alpha}-8(1+B) k_{1} k_{2} T e^{-i \alpha}+k_{1}^{3} T^{3} e^{-3 i \alpha}}{48} \\
& -\frac{3(1+B) k_{1}^{3} T^{2} e^{-2 i \alpha}+2(1+B)^{2} k_{1}^{3} T e^{-i \alpha}}{48} .
\end{aligned}
$$

Squaring (14) and (15), respectively, we get

$$
a_{2}^{2}=\frac{k_{1}^{2} T^{2} e^{-2 i \alpha}}{4}
$$

$$
\begin{aligned}
a_{3}^{2} & =\frac{4 k_{2}{ }^{2} T^{2} e^{-2 i \alpha}+(1+B)^{2} k_{1}{ }^{4} T^{2} e^{-2 i \alpha}-4(1+B) k_{1}{ }^{2} k_{2} T^{2} e^{-2 i \alpha}}{64} \\
& -\frac{2(1+B) k_{1}{ }^{4} T^{3} e^{-3 i \alpha}+4 k_{1}{ }^{2} k_{2} T^{3} e^{-3 i \alpha}+k_{1}{ }^{4} T^{4} e^{-4 i \alpha}}{64},
\end{aligned}
$$

and so by some simple computations, the determinant $T_{2}(2)$ can be written as

$$
\begin{aligned}
\left|a_{2}^{2}-a_{3}^{2}\right|= & \mid \frac{16 k_{1}^{2} T^{2} e^{-2 i \alpha}-(1+B)^{2} k_{1}^{4} T^{2} e^{-2 i \alpha}+2(1+B) k_{1}^{4} T^{3} e^{-3 i \alpha}-k_{1}^{4} T^{4} e^{-4 i \alpha}}{64} \\
& -\frac{4 k_{2}^{2} T^{2} e^{-2 i \alpha}+4(1+B) k_{1}^{2} k_{2} T^{2} e^{-2 i \alpha}-4 k_{1}^{2} k_{2} T^{3} e^{-3 i \alpha}}{64} \mid \\
= & \frac{\left|T^{2} e^{-2 i \alpha}\right|}{64} \mid 16 k_{1}^{2}+k_{1}^{4}\left(-(1+B)^{2}+2(1+B) T e^{-i \alpha}-T^{2} e^{-2 i \alpha}\right) \\
& -4 k_{2}^{2}+4 k_{1}^{2} k_{2}\left(-T e^{-i \alpha}+(1+B)\right) \mid \\
= & \frac{T^{2} e^{-2 i \alpha} \mid}{64} \mid 16 k_{1}^{2}+k_{1}^{4}\left(-(1+B)^{2}+2(1+B) T e^{-i \alpha}-T^{2} e^{-2 i \alpha}\right)- \\
& 4 k_{2}\left[k_{2}-\lambda k_{1}^{2}\right] \mid
\end{aligned}
$$


where $\lambda=-T e^{-i \alpha}+(1+B)$.

Consequently, by the triangle inequality

$\left|a_{2}^{2}-a_{3}^{2}\right| \leq \frac{T^{2}}{64}\left(16\left|k_{1}^{2}\right|+\left|k_{1}^{4}\right| \mid-(1+B)^{2}+2(1+B) T e^{-i \alpha}-\right.$

$\left.T^{2} e^{-2 i \alpha}|+4| k_{2}|| k_{2}-\lambda k_{1}^{2} \mid\right)$

which gives

$$
\begin{aligned}
& \left|2(1+B) T e^{-i \alpha}-(1+B)^{2}-T^{2} e^{-2 i \alpha}\right| \\
= & \left|e^{-i \alpha}\right|\left|2(1+B) T-(1+B)^{2} e^{i \alpha}-T^{2} e^{-i \alpha}\right| \\
= & \left|2(1+B) T-\cos \alpha\left((1+B)^{2}+T^{2}\right)-i \sin \alpha\left((1+B)^{2}-T^{2}\right)\right| \\
= & \sqrt{\left[2(1+B) T-\cos \alpha\left((1+B)^{2}+T^{2}\right)\right]^{2}+\left[-\sin \alpha\left((1+B)^{2}-T^{2}\right)\right]^{2}} .
\end{aligned}
$$

By Lemma 2,

$$
\left|k_{2}-\lambda k_{1}^{2}\right| \leq 2 \max \{1,|2 \lambda-1|\}
$$

where

$$
\begin{aligned}
|2 \lambda-1| & =\left|2\left(-T e^{-i \alpha}+(1+B)\right)-1\right| \\
& =|1+2 B-2 T \cos \alpha+2 T i \sin \alpha| \\
& =\sqrt{(1+2 B-2 T \cos \alpha)^{2}+(2 T \sin \alpha)^{2}} \\
& =\sqrt{(1+2 B)^{2}-4(1+2 B) T \cos \alpha+4 T^{2}} .
\end{aligned}
$$

Thus,

$$
\left|k_{2}-\lambda k_{1}^{2}\right| \leq 2 \max \left\{1, \sqrt{(1+2 B)^{2}-4(1+2 B) T \cos \alpha+4 T^{2}}\right\} .
$$

Applying Lemma 1 and using (21), from (20), we obtain

$$
\begin{aligned}
\left|a_{2}{ }^{2}-a_{3}{ }^{2}\right| \leq & \frac{T^{2}}{64}\left\{64+16 \sqrt{\left[2(1+B) T-\cos \alpha\left((1+B)^{2}+T^{2}\right)\right]^{2}+\left[-\sin \alpha\left((1+B)^{2}-T^{2}\right)\right]^{2}}\right. \\
& \left.+16 \sqrt{(1+2 B)^{2}-4(1+2 B) T \cos \alpha+4 T^{2}}\right\} .
\end{aligned}
$$

The result is sharp for the function given by $\left\{e^{i \alpha} \frac{z f^{\prime}(z)}{g(z)}-\delta-i \sin \alpha\right\} \frac{1}{t_{\alpha \delta}}=\frac{1+z}{1-z}$. This completes the proof of Theorem 1 .

Theorem 2 If $f \in S_{C}{ }^{*}(\alpha, \delta, A, B)$, then

$$
\begin{aligned}
\left|T_{3}(2)\right| \leq & \frac{T^{3}}{4608}\left\{48+16+2 \sqrt{64(1+B)^{2}-96(1+B) T \cos \alpha+36 T^{2}}\right. \\
& \left.\cdot\left[\frac{\left.2 \sqrt{\left[-3 B T+\cos \alpha\left(2\left(B^{2}-1\right)+T^{2}\right)\right]^{2}+\left[\sin \alpha\left(2\left(B^{2}-1\right)-T^{2}\right)\right.}\right]^{2}}{\sqrt{16(1+B)^{2}-24(1+B) T \cos \alpha+9 T^{2}}}\right]\right\} \\
& \cdot\left\{96+48+16 \sqrt{4(B+2)^{2}-12(B+2) T \cos \alpha+9 T^{2}}\right. \\
& \left.+16 \sqrt{\left[3(1+B) T-\cos \alpha\left((1+B)^{2}+2 T^{2}\right)\right]^{2}+\left[\sin \alpha\left((1+B)^{2}-2 T^{2}\right)\right]^{2}}\right\}
\end{aligned}
$$

where $T=(A-B) t_{\alpha \delta}$ and $t_{\alpha \delta}=\cos \alpha-\delta$. The inequality is sharp.

Proof. Upon simplification, the determinant $T_{3}(2)$ can be written as

$$
\begin{aligned}
\left|T_{3}(2)\right| & =\left|\left(a_{2}-a_{4}\right)\left(a_{2}^{2}-2 a_{3}^{2}+a_{2} a_{4}\right)\right| \\
& \leq\left|a_{2}-a_{4}\right|\left|a_{2}^{2}-2 a_{3}^{2}+a_{2} a_{4}\right| .
\end{aligned}
$$

Using (14) and (16) yield

$$
\begin{aligned}
a_{2}-a_{4}= & \frac{k_{1} T e^{-i \alpha}}{2}-\left[\frac{8 k_{3} T e^{-i \alpha}+6 k_{1} k_{2} T^{2} e^{-2 i \alpha}-8(1+B) k_{1} k_{2} T e^{-i \alpha}+k_{1}^{3} T^{3} e^{-3 i \alpha}}{48}\right. \\
& \left.-\frac{3(1+B) k_{1}^{3} T^{2} e^{-2 i \alpha}+2(1+B)^{2} k_{1}^{3} T e^{-i \alpha}}{48}\right],
\end{aligned}
$$

and so

$$
\begin{aligned}
\left|a_{2}-a_{4}\right| & =\mid \frac{24 k_{1} T e^{-i \alpha}+8(1+B) k_{1} k_{2} T e^{-i \alpha}-6 k_{1} k_{2} T^{2} e^{-2 i \alpha}-8 k_{3} T e^{-i \alpha}}{48} \\
& -\frac{2(1+B)^{2} k_{1}^{3} T e^{-i \alpha}+3(1+B) k_{1}^{3} T^{2} e^{-2 i \alpha}-k_{1}^{3} T^{3} e^{-3 i \alpha} \mid}{48} \mid \\
& =\frac{\left|T e^{-i \alpha}\right|}{48} \mid 24 k_{1}-8 k_{3}+k_{1}\left[k_{2}\left(8(1+B)-6 T e^{-i \alpha}\right)\right. \\
& \left.-k_{1}^{2}\left(-3(1+B) T e^{-i \alpha}+2(1+B)^{2}+T^{2} e^{-2 i \alpha}\right)\right] \mid \\
& =\frac{\left|T e^{-i \alpha}\right|}{48} \mid 24 k_{1}-8 k_{3}+k_{1}\left(8(1+B)-6 T e^{-i \alpha}\right) \\
& \cdot\left[k_{2}-k_{1}^{2} \frac{\left(-3(1+B) T e^{-i \alpha}+2(1+B)^{2}+T^{2} e^{-2 i \alpha}\right)}{\left(8(1+B)-6 T e^{-i \alpha}\right)}\right] \mid \\
& =\frac{\left|T e^{-i \alpha}\right|}{48}\left|24 k_{1}-8 k_{3}+k_{1}\left(8(1+B)-6 T e^{-i \alpha}\right)\left[k_{2}-\gamma k_{1}^{2}\right]\right|
\end{aligned}
$$

where $\gamma=\frac{-3(1+B) T e^{-i \alpha}+2(1+B)^{2}+T^{2} e^{-2 i \alpha}}{8(1+B)-6 T e^{-i \alpha}}$.

Consequently, by the triangle inequality

$$
\begin{aligned}
& \left|a_{2}-a_{4}\right| \leq \frac{\left|T e^{-i \alpha}\right|}{48}\left(24\left|k_{1}\right|+8\left|k_{3}\right|+\left|k_{1}\right| \mid 8(1+B)-\right. \\
& \left.6 T e^{-i \alpha}|| k_{2}-\gamma k_{1}^{2} \mid\right) .
\end{aligned}
$$

By using the similar approach as in the proof of Theorem 1 , from (23), we get

$$
\begin{aligned}
\left|a_{2}-a_{4}\right| & \leq \frac{T}{48}\left\{48+16+2\left(\sqrt{64(1+B)^{2}-96(1+B) T \cos \alpha+36 T^{2}}\right)\right. \\
& \left.\cdot\left[\frac{\left.2 \sqrt{\left[-3 B T+\cos \alpha\left(2\left(B^{2}-1\right)+T^{2}\right)\right]^{2}+\left[\sin \alpha\left(2\left(B^{2}-1\right)-T^{2}\right)\right.}\right]}{\sqrt{16(1+B)^{2}-24(1+B) T \cos \alpha+9 T^{2}}}\right]\right\} .
\end{aligned}
$$

Equations (14) - (18) together yield 
$\left|a_{2}{ }^{2}-2 a_{3}{ }^{2}+a_{2} a_{4}\right|=\mid \frac{k_{1}^{2} T^{2} e^{-2 i \alpha}}{4}-2\left[\frac{4 k_{2}^{2} T^{2} e^{-2 i \alpha}+4 k_{1}^{2} k_{2} T^{3} e^{-3 i \alpha}-4(1+B) k_{1}^{2} k_{2} T^{2} e^{-2 i \alpha}+k_{1}^{4} T^{4} e^{-4 i \alpha}}{64}\right.$ $\left.-\frac{2(1+B) k_{1}^{4} T^{3} e^{-3 i \alpha}+(1+B)^{2} k_{1}^{4} T^{2} e^{-2 i \alpha}}{64}\right]+\frac{8 k_{1} k_{3} T^{2} e^{-2 i \alpha}+6 k_{1}^{2} k_{2} T^{3} e^{-3 i \alpha}}{96}$ $-\frac{8(1+B) k_{1}^{2} k_{2} T^{2} e^{-2 i \alpha}+k_{1}^{4} T^{4} e^{-4 i \alpha}-3(1+B) k_{1}^{4} T^{3} e^{-3 i \alpha}+2(1+B)^{2} k_{1}^{4} T^{2} e^{-2 i \alpha}}{96} \mid$ $=\mid \frac{24 k_{1}^{2} T^{2} e^{-2 i \alpha}-2 k_{1}^{4} T^{4} e^{-4 i \alpha}-(1+B)^{2} k_{1}^{4} T^{2} e^{-2 i \alpha}+3(1+B) k_{1}^{4} T^{3} e^{-3 i \alpha}}{96}$ $+\frac{4(1+B) k_{1}^{2} k_{2} T^{2} e^{-2 i \alpha}-6 k_{1}^{2} k_{2} T^{3} e^{-3 i \alpha}-12 k_{2}^{2} T^{2} e^{-2 i \alpha}+8 k_{1} k_{3} T^{2} e^{-2 i \alpha}}{96}$.

Further, by arranging the terms, we have

$$
\begin{aligned}
\left|a_{2}^{2}-2 a_{3}^{2}+a_{2} a_{4}\right| & =\mid \frac{T^{2} e^{-2 i \alpha}\left[24 k_{1}{ }^{2}-12 k_{2}{ }^{2}+k_{1}{ }^{4}\left(3(1+B) T e^{-i \alpha}-(1+B)^{2}-2 T^{2} e^{-2 i \alpha}\right)\right.}{96} \\
& +\frac{8 k_{1}\left[k_{3}-k_{2} k_{1} \frac{\left(-4(1+B)+6 T e^{-i \alpha}\right)}{8}\right]}{96}|| \\
& =\mid \frac{T^{2} e^{-2 i \alpha}\left[24 k_{1}{ }^{2}-12 k_{2}{ }^{2}+k_{1}{ }^{4}\left(3(1+B) T e^{-i \alpha}-(1+B)^{2}-2 T^{2} e^{-2 i \alpha}\right)\right.}{96} \\
& +\frac{\left.8 k_{1}\left[k_{3}-\chi k_{2} k_{1}\right]\right]}{96} \mid
\end{aligned}
$$

where $\chi=\frac{-4(1+B)+6 T e^{-i \alpha}}{8}$.

Applying the triangle inequality, (25) yields

$$
\begin{aligned}
\mid a_{2}^{2}-2 a_{3}^{2}+ & a_{2} a_{4} \mid \leq \frac{\left|T^{2} e^{-2 i \alpha}\right|}{96}\left(24\left|k_{1}^{2}\right|+12\left|k_{2}^{2}\right|+\left|k_{1}^{4}\right| \mid 3(1+B) T e^{-i \alpha}-\right. \\
& \left.-2 T^{2} e^{-2 i \alpha}|+8| k_{1}|| k_{3}-\chi k_{2} k_{1} \mid\right) .
\end{aligned}
$$

By using the similar approach as in the proof of Theorem 1 , from (26), we get

$$
\begin{aligned}
\left|a_{2}{ }^{2}-2 a_{3}{ }^{2}+a_{2} a_{4}\right| \leq & \frac{T^{2}}{96}\left\{96+48+16 \sqrt{4(B+2)^{2}-12(B+2) T \cos \alpha+9 T^{2}}\right. \\
& \left.+16 \sqrt{\left[\left[3(1+B) T-\cos \alpha\left((1+B)^{2}+2 T^{2}\right)\right]^{2}+\left[\sin \alpha\left((1+B)^{2}-2 T^{2}\right)\right]^{2}\right.}\right\} .
\end{aligned}
$$

Hence, using (24) and (27), we obtain

$$
\begin{aligned}
\left|T_{3}(2)\right| & \leq \frac{T^{3}}{4608}\left\{48+16+2 \sqrt{64(1+B)^{2}-96(1+B) T \cos \alpha+36 T^{2}}\right. \\
& \left.\cdot\left[\frac{2 \sqrt{\left[-3 B T+\cos \alpha\left(2\left(B^{2}-1\right)+T^{2}\right)\right]^{2}+\left[\sin \alpha\left(2\left(B^{2}-1\right)-T^{2}\right)\right]^{2}}}{\sqrt{16(1+B)^{2}-24(1+B) T \cos \alpha+9 T^{2}}}\right]\right\} \\
& \cdot\left\{96+48+16 \sqrt{4(B+2)^{2}-12(B+2) T \cos \alpha+9 T^{2}}\right. \\
& \left.+16 \sqrt{\left[3(1+B) T-\cos \alpha\left((1+B)^{2}+2 T^{2}\right)\right]^{2}+\left[\sin \alpha\left((1+B)^{2}-2 T^{2}\right)\right]^{2}}\right\} .
\end{aligned}
$$

The result is sharp for the function given by $\left\{e^{i \alpha} \frac{z f^{\prime}(z)}{g(z)}-\delta-i \sin \alpha\right\} \frac{1}{t_{\alpha \delta}}=\frac{1+z}{1-z}$. This completes the proof of Theorem 2 .

Theorem 3 If $f \in S_{C}^{*}(\alpha, \delta, A, B)$, then

$\left|T_{3}(1)\right| \leq 1+2 T^{2}+\left(\frac{T^{2} \sqrt{B^{2}-2 B T \cos \alpha+T^{2}} \sqrt{B^{2}+6 B T \cos \alpha+9 T^{2}}}{4}\right)$ where $T=(A-B) t_{\alpha \delta}$ and $t_{\alpha \delta}=\cos \alpha-\delta$. The inequality is sharp.

Proof. Expanding the determinant $T_{3}(1)$, we get

$$
\begin{aligned}
\left|T_{3}(1)\right| & =\left|1-2 a_{2}{ }^{2}+2 a_{2}{ }^{2} a_{3}-a_{3}^{2}\right| \\
& \leq 1+2\left|a_{2}{ }^{2}\right|+\left|a_{3}\right|\left|a_{3}-2 a_{2}{ }^{2}\right| .
\end{aligned}
$$

By using Lemma 1, from (17) yields

$$
\left|a_{2}^{2}\right|=\left|\frac{k_{1}^{2} T^{2} e^{-2 i \alpha}}{4}\right| \leq T^{2}
$$

and by arranging the terms in (15), we have

$$
\begin{aligned}
\left|a_{3}\right| & =\left|\frac{2 k_{2} T e^{-i \alpha}+k_{1}^{2} T^{2} e^{-2 i \alpha}-(1+B) k_{1}^{2} T e^{-i \alpha}}{8}\right| \\
& =\left|\frac{2 T e^{-i \alpha}\left[k_{2}-\frac{k_{1}^{2}}{2}\left((1+B)-T e^{-i \alpha}\right)\right]}{8}\right| \\
& =\left|\frac{T e^{-i \alpha}\left[k_{2}-\mu k_{1}^{2}\right]}{4}\right|
\end{aligned}
$$

where $\mu=\frac{(1+B)-T e^{-i \alpha}}{2}$.

Following the same approach as in Theorem 1, thus

$$
\left|a_{3}\right| \leq \frac{T \sqrt{B^{2}-2 B T \cos \alpha+T^{2}}}{2} .
$$

Equations (15) and (17) together yield

$$
\begin{aligned}
\left|a_{3}-2 a_{2}{ }^{2}\right| & =\left|\frac{2 k_{2} T e^{-i \alpha}+k_{1}^{2} T^{2} e^{-2 i \alpha}-(1+B) k_{1}^{2} T e^{-i \alpha}}{8}-\frac{2 k_{1}^{2} T^{2} e^{-2 i \alpha}}{4}\right| \\
& =\left|\frac{2 T e^{-i \alpha}\left[k_{2}-\frac{k_{1}^{2}}{2}\left((1+B)+3 T e^{-i \alpha}\right)\right]}{8}\right| \\
& =\left|\frac{T e^{-i \alpha}\left[k_{2}-v k_{1}^{2}\right]}{4}\right|
\end{aligned}
$$


where

$$
v=\frac{(1+B)+3 T e^{-i \alpha}}{2} .
$$

Following the same approach as in Theorem 1, we find that

$$
\left|a_{3}-2 a_{2}^{2}\right| \leq \frac{T \sqrt{B^{2}+6 B T \cos \alpha+9 T^{2}}}{2}
$$

Hence, by using Lemma 1, (28), (29) and (31), we obtain $\left|T_{3}(1)\right| \leq 1+2 T^{2}+\left(\frac{T^{2} \sqrt{B^{2}-2 B T \cos \alpha+T^{2}} \sqrt{B^{2}+6 B T \cos \alpha+9 T^{2}}}{4}\right)$. The result is sharp for the function given by $\left\{e^{i \alpha} \frac{z f^{\prime}(z)}{g(z)}-\delta-i \sin \alpha\right\} \frac{1}{t_{\alpha \delta}}=\frac{1+z}{1-z}$. This completes the proof of Theorem 3.

Choosing and substituting the specific values for the parameters into the results of Theorem 1, Theorem 2 and Theorem 3 we obtain the following corollaries. Corollary 1

a) For $f \in S_{C}{ }^{*}(0,0,1,-1)$, we get $\left|a_{2}^{2}-a_{3}^{2}\right| \leq 13$.

b) For $f \in S_{C}{ }^{*}(0,0,1,-1)$, we get $\left|T_{3}(2)\right| \leq 84$.

c) For $f \in S_{C}^{*}(0,0,1,-1)$, we get $\left|T_{3}(1)\right| \leq 24$.

\section{Corollary 2}

a) For $f \in S_{C}^{*}(0, \delta, 1,-1)$, we get

$\left|a_{2}^{2}-a_{3}{ }^{2}\right| \leq(1-\delta)^{2}\left[4+\sqrt{16(1-\delta)^{4}}+\sqrt{1+8(1-\delta)+16(1-\delta)^{2}}\right]$.

b) For $f \in S_{C}{ }^{*}(0, \delta, 1,-1)$, we get

$$
\begin{aligned}
\left|T_{3}(2)\right| & \leq \frac{8(1-\delta)^{3}}{4608}\left\{48+16+24(1-\delta)\left[\frac{2\left(3(1-\delta)+2(1-\delta)^{2}\right)}{3(1-\delta)}\right]\right\} \\
& \bullet\left\{96+48+32 \sqrt{1-6(1-\delta)+9(1-\delta)^{2}}+128(1-\delta)^{2}\right\} .
\end{aligned}
$$

c) For $f \in S_{C}{ }^{*}(0, \delta, 1,-1)$, we get

$\left|T_{3}(1)\right| \leq 1+8(1-\delta)^{2}+(1-\delta)^{2} \sqrt{1+4(1-\delta)+4(1-\delta)^{2}} \sqrt{1-12(1-\delta)+36(1-\delta)^{2}}$.

\section{Corollary 3}

a) For $f \in S_{C}{ }^{*}(0,0, A, B)$, we get

$$
\begin{aligned}
\left|a_{2}^{2}-a_{3}{ }^{2}\right| \leq & \frac{(A-B)^{2}}{4}\left\{4+\sqrt{\left[2(1+B)(A-B)-(1+B)^{2}-(A-B)^{2}\right]^{2}}\right. \\
& \left.+\sqrt{(1+2 B)^{2}-4(1+2 B)(A-B)+4(A-B)^{2}}\right\} .
\end{aligned}
$$

b) For $f \in S_{C}^{*}(0,0, A, B)$, we get

$$
\begin{aligned}
\left|T_{3}(2)\right| \leq & \frac{(A-B)^{3}}{4608}\left\{48+16+2 \sqrt{64(1+B)^{2}-96(1+B)(A-B)+36(A-B)^{2}}\right. \\
& \left.\cdot\left[\frac{2 \sqrt{\left[-3 B(A-B)+2\left(B^{2}-1\right)+(A-B)^{2}\right]^{2}}}{\sqrt{16(1+B)^{2}-24(1+B)(A-B)+9(A-B)^{2}}}\right]\right\} \\
& \cdot\left\{96+48+16 \sqrt{4(B+2)^{2}-12(B+2)(A-B)+9(A-B)^{2}}\right. \\
& \left.+16 \sqrt{\left[3(1+B)(A-B)-(1+B)^{2}-2(A-B)^{2}\right]^{2}}\right\} .
\end{aligned}
$$

c) For $f \in S_{C}^{*}(0,0, A, B)$, we get

$$
\begin{aligned}
\left|T_{3}(1)\right| \leq & 1+2(A-B)^{2}+\frac{1}{4}\left[(A-B)^{2} \sqrt{B^{2}-2 B(A-B)+(A-B)^{2}}\right. \\
& \left.\cdot \sqrt{B^{2}+6 B(A-B)+9(A-B)^{2}}\right] .
\end{aligned}
$$

Corollaries 1, 2 and 3 show the coefficient bounds for the Toeplitz determinants for the subclasses introduced by El-Ashwah and Thomas (1987), Halim (1991) and Dahhar and Janteng (2009), respectively. The inequalities in Corollary 1 coincide with the results of Ali et al. (2018) for the class of starlike functions $S^{*}$. Notice that the result of coefficient bounds for the Toeplitz determinants for the class of starlike functions $S^{*}$ and the class of starlike functions with respect to conjugate points $S_{C}{ }^{*}$ are shown to be equivalent.

\section{CONCLUSION}

In this paper, we have obtained the coefficient bounds for the Toeplitz determinants $T_{2}(2), T_{3}(1)$ and $T_{3}(2)$ for the class $S_{C}{ }^{*}(\alpha, \delta, A, B)$. It is shown that by considering specific values for the parameters $\alpha, \delta, A$ and $B$ the results obtained can be reduced to the results for some existing subclasses.

\section{ACKNOWLEDGEMENTS}

The authors would like to thank Universiti Teknologi MARA for her support towards the completion of this paper.

\section{REFERENCES}

Al-Khafaji, S.N., Al-Fayadh, A., Hussain, A.H. \& Abbas, S. A. 2020, November. Toeplitz determinant whose its entries are the coefficients for class of Non-Bazilevič functions. In Journal of Physics: Conference Series. IOP Publishing. 1660(1): 012091

Ali, M.F., Thomas, D.K. \& Vasudevarao, A. 2018. Toeplitz determinants whose elements are the coefficients of 
analytic and univalent functions. Bulletin of the Australian Mathematical Society 97(2): 253-264.

Dahhar, S.A.F.M. \& Janteng, A. 2009. A subclass of starlike functions with respect to conjugate points. International Mathematical Forum 4(28): 1373-1377.

Duren, P.L. 1983. Univalent Functions. New York-BerlinHeidelberg-Tokyo: Springer.

Efraimidis, I. 2016. A generalization of Livingston's coefficient inequalities for functions with positive real part. Journal of Mathematical Analysis and Applications 435(1): 369-379.

El-Ashwah, R.M. \& Thomas, D.K. 1987. Some subclasses of close-to-convex functions. Journal of the Ramanujan Mathematical Society 2(1): 85-100.

Halim, S.A. 1991. Functions starlike with respect to other points. International Journal of Mathematics and Mathematical Sciences 14(3): 451-456.

Pommerenke, C. 1967. On the Hankel determinants of univalent functions. Mathematika 14(1): 108-112.

Pommerenke, C. 1966. On the coefficients and Hankel determinants of univalent functions. Journal of the London Mathematical Society 1(1): 111-122.

Radhika, V., Jahangiri, J.M., Sivasubramanian, S. \& Murugusundaramoorthy, G. 2018. Toeplitz matrices whose elements are coefficients of Bazilevič functions. Open Mathematics 16(1): 1161-1169.

Radhika, V., Sivasubramanian, S., Murugusundaramoorthy, G. \& Jahangiri, J.M. 2016. Toeplitz matrices whose elements are the coefficients of functions with bounded boundary rotation. Journal of Complex Analysis 2016: 4960704.

Ramachandran, C. \& Kavitha, D. 2017. Toeplitz determinant for some subclasses of analytic functions. Global Journal of Pure and Applied Mathematics 13(2): 785-793.
Sivasubramanian, S., Govindaraj, M. \& Murugusundaramoorthy, G. 2016. Toeplitz matrices whose elements are the coefficients of analytic functions belonging to certain conic domains. International Journal of Pure and Applied Mathematics 109(10): 39-49.

Srivastava, H.M., Ahmad, Q.Z., Khan, N., Khan, N. \& Khan, B. 2019. Hankel and Toeplitz determinants for a subclass of q-starlike functions associated with a general conic domain. Mathematics 7(2): 181.

Thomas, D.K. \& Halim, S.A. 2016. Toeplitz matrices whose elements are the coefficients of starlike and close-to-convex functions. Bulletin of the Malaysian Mathematical Sciences Society 40(4): 1781-1790.

Ye, K. \& Lim, L.H. 2016. Every matrix is a product of Toeplitz matrices. Foundations of Computational Mathematics 16(3): 577-598.

Wahid, N.H.A.A., Mohamad, D. \& Cik Soh, S. 2015. On a subclass of tilted starlike functions with respect to conjugate points. Discovering Mathematics 37(1): 1-6.

Zhang, H.Y., Srivastava, R. \& Tang, H. 2019. Third-order Hankel and Toeplitz determinants for starlike functions connected with the sine function. Mathematics 7(5): 404.

Faculty of Computer and Mathematical Sciences

Universiti Teknologi MARA

40450 Shah Alam, Selangor Darul Ehsan

Malaysia

*Corresponding author; email: daud@tmsk.uitm.edu.my

Received: 29 January 2021

Accepted: 24 March 2021 